\title{
Chapter 36 \\ Regulation of Vacuole-Mediated Programmed Cell Death During Innate Immunity and Reproductive Development in Plants
}

\author{
Tomoko Koyano, Takamitsu Kurusu, Shigeru Hanamata, \\ and Kazuyuki Kuchitsu
}

\begin{abstract}
Programmed cell death (PCD), organized destruction of cells, is essential in development, maintenance of cellular homeostasis, and innate immunity in multicellular organisms. In most angiosperms, development of male and female organs involves spatial and temporal regulation of PCD. The tapetum, the innermost layer of the anther, provides both nutrient and lipid components to developing microspores, and has been proposed to be degraded by PCD during the later stages of pollen maturation. Plants lack homologues of most apoptosis-related genes in animals and have evolved specific mechanisms for PCD. PCD is also a crucial event in plant immune responses against microbial infection that prevents the spread of pathogens. Recent live cell imaging techniques have revealed the dynamic features and significant roles of the vacuole during defense responses and PCD. Disintegration or collapse of the vacuolar membrane has been suggested to trigger the final step of
\end{abstract}

T. Koyano and T. Kurusu contributed equally to this work.

T. Koyano $\bullet$ S. Hanamata

Department of Applied Biological Science, Tokyo University of Science,

2641 Yamazaki, Noda, Chiba 278-8510, Japan

T. Kurusu

Department of Applied Biological Science, Tokyo University of Science,

2641 Yamazaki, Noda, Chiba 278-8510, Japan

Research Institute for Science and Technology, Tokyo University of Science, 2641 Yamazaki, Noda, Chiba 278-8510, Japan

School of Bioscience and Biotechnology, Tokyo University of Technology, 1404-1 Katakura, Hachioji, Tokyo 192-0982, Japan

K. Kuchitsu ( $\square)$

Department of Applied Biological Science, Tokyo University of Science,

2641 Yamazaki, Noda, Chiba 278-8510, Japan

Research Institute for Science and Technology, Tokyo University of Science,

2641 Yamazaki, Noda, Chiba 278-8510, Japan

e-mail: kuchitsu@rs.noda.tus.ac.jp 
PCD in several cell types. We here overview spatiotemporal dynamic changes of the vacuole triggered by signals from pathogens and comparatively discuss PCD during innate immunity and reproductive development in plants.

Keywords Innate immunity $\bullet$ Programmed cell death $\bullet$ Reproductive development

- Tapetum • Vacuole

\subsection{Programmed Cell Death in Plants}

Programmed cell death (PCD) is a genetically regulated process of cellular suicide that is well known to play a fundamental role in a wide variety of developmental and physiological functions in multicellular organisms (Bozhkov and Lam 2011; Fuchs and Steller 2011; Teng et al. 2011). In plants, PCD plays a critical role in the control of developmental processes such as xylogenesis, embryogenesis, pollen maturation, seed development, seed germination, and leaf senescence, as well as various stress responses including innate immunity against pathogen attack (Pennell and Lamb 1997). Reproductive development in angiosperms involves PCD in a variety of cells in reproductive organs, such as reproductive primordium abortion, style transmitting tissue, nonfunctional megaspores, synergids, antipodals, endosperm, anther tapetum, and abortive pollen in male sterility (Greenberg 1996; Pennell and Lamb 1997; Wei et al. 2002). Plants lack homologues of most apoptosis-related genes in animals and have evolved specific mechanisms for PCD. We here comparatively discuss mechanisms for PCD in innate immunity and reproductive development with special reference to the roles of the vacuole.

\subsection{PCD in Plant Immunity Against Pathogen Attack}

Plants lack immune systems based on antibodies or phagocytosis. Instead, they have evolved multiple layers of active defense responses including the deliberate production of reactive oxygen species (ROS), pathogenesis-related (PR) proteins, and antimicrobial secondary metabolites called phytoalexins. The dynamic reorganization of plant cells is triggered at the site of infection and often accompanies localized PCD, known as the hypersensitive response (HR), which is effective in preventing the spread of pathogens (Heath 2000; Mur et al. 2008).

The plant cells show different characteristics from animal cells, most notably the presence of the cell wall, the plastids/chloroplasts, and the vacuole, all of which play crucial roles in the regulation of plant immunity and PCD. This difference suggests that execution of PCD takes place with different morphological features from typical animal PCD such as apoptosis. Cellular morphological changes in animal cells undergoing apoptosis, including cell shrinkage and nuclear fragmentation, are followed by the fragmentation of cells and formation of apoptotic bodies, which are 
then phagocytosed. Although the similarities and differences between PCD in plants and animals have been extensively discussed (Cacas 2010; Reape and McCabe 2010), the mechanisms for execution and regulation of plant PCD including HR still largely unclear.

Cell biological aspects of immune responses accompanying PCD have been studied in various experimental systems using a combination of plants and microbes. A major experimental approach is immunostaining of plant tissues infected with microbes (Kobayashi et al. 1994; Skalamera and Heath 1996; Kobayashi et al. 1997). However, the deformation of endomembrane systems by chemical fixation with this technique should be noted. Recently, green fluorescent protein (GFP)-based in vivo imaging has allowed time-sequential observations of the endomembrane systems in plant-microbe interactions (Takemoto et al. 2003; Koh et al. 2005).

The execution of cell death in a regulated fashion should accompany dynamic reorganization of the cellular architecture. In plants, cellular morphological changes are often governed by cytoskeletons such as actin microfilaments and microtubules, as well as the vacuole, an organelle occupying most of the cell volume. In recent years, the rapid development of live cell imaging techniques has provided novel aspects on the dynamics of intracellular structures. We here focus on the dynamics of the vacuole and discuss its functional significance in innate immunity and PCD.

\subsubsection{Tobacco BY-2 Cells as an Excellent Model System to Study Immune Responses Accompanying Localized Plant PCD}

Plant cell cultures are useful simple model systems for the monitoring of cellular events. Defense responses including intracellular reorganization and gene expression upon fungal infection are basically similar between cultured cells and in planta (Gross et al. 1993). Treatment of cultured cells with purified signal molecules from pathogens can mimic most defense responses, including the expression of defenserelated genes, as effectively as microbial infections (Lecourieux et al. 2002; Kadota et al. 2004a; Kadota and Kuchitsu 2006).

Treatment of tobacco (Nicotiana tabacum) BY-2 cells with cryptogein, a protein from the oomycete Phytophthora cryptogea, induces various immune responses such as membrane potential changes, ion fluxes, biphasic ROS production, and MAP kinase activation in a cell cycle-dependent manner (Kadota et al. 2005; Kadota et al. 2006), followed by cell-cycle arrest and PCD (Kadota et al. 2004b; Ohno et al. 2011). The slow prolonged phase, not the rapid transient phase, of ROS production shows strong correlation with downstream events including expression of defenserelated genes and PCD (Kadota et al. 2005; Kadota et al. 2006).

NADPH oxidase-mediated deliberate ROS production has been suggested to play a crucial role in triggering and regulating PCD (Torres et al. 2005; Suzuki et al. 2011). Respiratory burst oxidase homologue (Rboh) proteins show ROS-producing activity synergistically activated by binding of $\mathrm{Ca}^{2+}$ to their EF-hand motifs and 

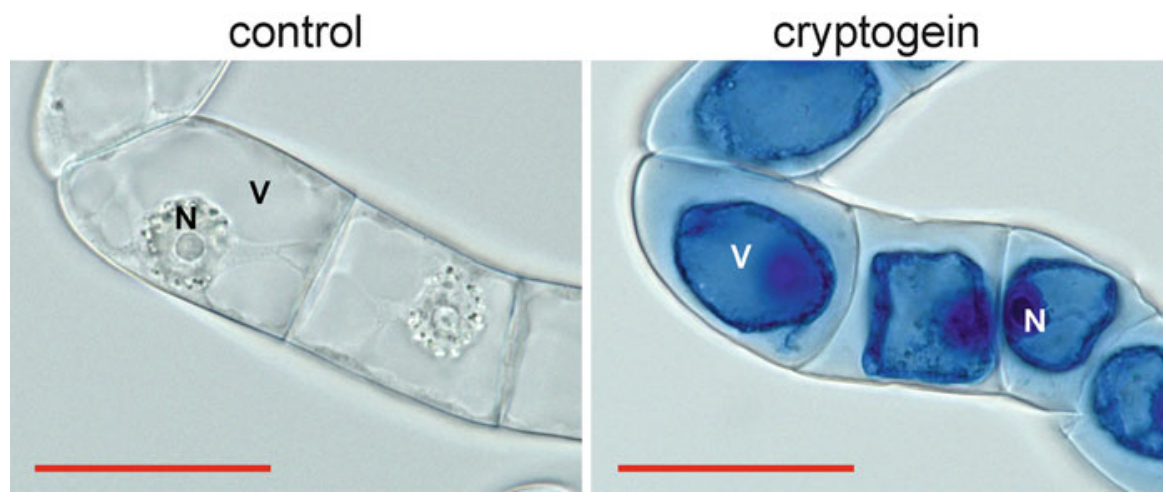

Fig. 36.1 Cryptogein-induced programmed cell death in suspension-cultured tobacco BY-2 cells. Three-day-old cultured BY-2 cells were treated with $1 \mu \mathrm{M}$ cryptogein or distilled water (control) for $24 \mathrm{~h}$. Dead cells were stained with Evans blue $24 \mathrm{~h}$ after cryptogein treatment (Higaki et al. 2007). $V$ vacuole, $N$ nucleus. Bar $50 \mu \mathrm{m}$.

protein phosphorylation (Ogasawara et al. 2008; Takeda et al. 2008; Kimura et al. 2012). Potato StRBOHB has been shown to be activated by phosphorylation by calcium-dependent protein kinases StCDPK4 and StCDPK5 (Kobayashi et al. 2007). Arabidopsis AtRbohF has recently been shown to bind CIPK26, a protein kinase activated by binding of calcineurin $\mathrm{B}$-like $\mathrm{Ca}^{2+}$ sensor proteins CBLs, in planta (Kimura et al. 2013), and be activated in the presence of CBL1/CBL9 and CIPK26 (Drerup et al. 2013).

Transgenic tobacco BY-2 cell lines expressing the GFP-markers for vacuolar membranes (VM; Kutsuna and Hasezawa 2002) have been effective in monitoring dynamic changes in vacuoles during cryptogein-induced defense responses in vivo (Higaki et al. 2007).

\subsubsection{Regulation of Vacuole-Mediated PCD in Innate Immunity}

The plant vacuole is an organelle that occupies most of the cell volume and contains many hydrolytic enzymes for digestive processes, similar to lysosomes in animal cells. The vacuole performs various functions essential for plant growth, development, and adaptation to both abiotic and biotic stresses (Marty 1999).

Disintegration or collapse of the VM has been suggested to trigger the final step of PCD in several cell types (Jones 2001; Hara-Nishimura and Hatsugai 2011; Higaki et al. 2011). At the final stage of cryptogein-induced PCD in tobacco BY-2 cells, disintegration of the VM is followed by the irreversible loss of plasma membrane integrity and cell shrinkage (Higaki et al. 2007; Fig. 36.1). Tobacco mosaic virus-induced hypersensitive cell death in tobacco leaves involves vacuolar rupture, in which a vacuolar-localized protease called vacuolar processing enzyme 
(VPE) exhibiting caspase-1-like activity is involved (Hatsugai et al. 2004, 2006). Disintegration of the VM or vacuolar collapse needs to be strictly regulated to accomplish PCD with appropriate timing. Another mechanism involving fusion of the VM with the plasma membrane, resulting in the discharge of vacuolar antibacterial proteins to the outside of the cells where bacteria proliferate, has also been proposed for biotrophic bacteria-induced hypersensitive cell death (Hatsugai et al. 2009).

Cryptogein-induced PCD in BY-2 cells accompanies dynamic reorganization of the vacuole before the execution of cell death (Higaki et al. 2008). Cryptogein induces decrease in the transvacuolar strands (TVS), tubular regions of the cytoplasm connecting the nucleus to the cell periphery, and formation of a spherical intravacuolar structure called the 'bulb' (Higaki et al. 2007) that has been observed in a wide range of plant tissues (Saito et al. 2002). The bulb-like structure could be derived from the excess VM comprising the TVS. At the later stage of the PCD, the bulb-like structure disappears and the structure of the large central vacuole becomes simpler. Architecture of the vacuole is governed by actin filaments. Indeed, an actin polymerization inhibitor facilitates both the disappearance of the bulb-like VM structures and induction of PCD (Higaki et al. 2007). These findings suggest that the elicitor-induced reorganization of actin microfilaments followed by modification of the vacuolar structure to induce VM disintegration plays a key role in induction of the PCD.

\subsection{Vacuole-Mediated PCD in Plant Vegetative Development}

Vacuolar collapse plays a critical role at the final stage of execution of PCD during differentiation of the tracheary element in Zinnia elegans (Kuriyama 1999; Obara et al. 2001). PCD in the inner integument cell layers of developing Arabidopsis seeds also involves vacuolar rupture, in which a vacuolar-localized protease called VPE exhibiting caspase-1-like activity is involved (Nakaune et al. 2005).

The structural simplification of vacuoles has been commonly observed in various PCD processes including tracheary element differentiation (Obara et al. 2001), gibberellin-mediated PCD in central aleurone cells (Guo and Ho 2008), embryogenesis in a gymnosperm (Smertenko et al. 2003), and leaf formation in a lace plant (Gunawardena 2008). Decreases in the number of TVS and VM reorganization were confirmed in the process of developmental PCD in the lace plant Aponogeton madagascariensis (Wright et al. 2009), which suggests a general role of VM reorganization in vacuolar rupture-mediated PCD in plants. 


\subsection{PCD in Plant Reproductive Development}

During plant reproduction, proper induction of PCD is prerequisite for reproductive success. Failure in PCD often results in sterility. In most angiosperms, development of male and female organs involves spatial and temporal regulation of PCD. For example, tapetal cells, the innermost layer of the anther, supply fundamental nutrients necessary for normal pollen development. Shortly after the microspore release from the tetrad and before mitosis, tapetal cells begin to degenerate by PCD. In female gamete development within the ovules, a typically diploid megaspore mother cell undergoes meiosis, giving four megaspores, and three of these megaspores undergo PCD.

Besides development of reproductive organs, PCD also has an important role in fertilization. For example, in self-incompatibility response in Papaver, an incompatible pollen tube is stopped by interactions with the pistil $S$-determinant by PCD (Bosch and Franklin-Tong 2008). In this way, PCD plays important roles in multiple steps of normal reproduction in plants from development of gametes to fertilization.

\subsubsection{PCD as a Key System for Tapetum Degradation During Pollen Development}

During pollen development, the tapetum is degraded to supply metabolites, nutrients, and sporopollenin precursors to developing microspores. Defects in the degradation of the tapetum cause the abnormal formation of the pollen coat and pollen grains and result in severe male sterility (Ku et al. 2003; Li et al. 2006; Zhang et al. 2008; Ariizumi and Toriyama 2011). The degradation of the tapetum is highly regulated and exhibits the hallmark features of PCD such as cell shrinkage, condensation of chromatin, swelling of the endoplasmic reticulum (ER), and persistence of the mitochondria (Rogers et al. 2005) as well as nuclear fragmentation (Wang et al. 1999; Vardar and Unal 2012).

The correct timing of tapetal PCD is important for normal pollen development. The initiation signal for tapetal PCD has been proposed to commence as early as the tetrad stage (Kawanabe et al. 2006). The ms 1 mutant showed delayed tapetal breakdown and a switch from PCD degradation to necrotic-based breakdown (VizcayBarrena and Wilson 2006). A rice mutant tapetal degeneration retardation (Ostdr) shows significantly delayed tapetal breakdown and PCD, resulting in the failure of pollen wall deposition and subsequent microspore degeneration ( $\mathrm{Li}$ et al. 2006; Zhang et al. 2008). Degradation of the tapetum was also regulated by a plant hormone gibberellin (Cheng et al. 2004; Aya et al. 2009). 


\subsubsection{Regulation of Vacuole-Mediated PCD in Tapetum Degradation}

Abnormal vacuolization in the tapetum during the tetrad stage causes abnormal tapetal PCD and results in male sterility (Wan et al. 2010). The vacuoles in the tapetum may supply tetrad wall-degrading enzymes before their secretion into the anther locules (Wu and Yang 2005). These results suggest important roles of tapetal vacuoles during anther development and PCD.

In the tapetum of Lathyrus undulatus L. at the vacuolated microspore stage, rupture of the VM and vacuolar collapse is induced, followed by possible release of hydrolytic enzymes and the degradation of cell components (Vardar and Unal 2012). Interestingly, the increment of VPE in the Arabidopsis anther has also been reported (Hatsugai et al. 2006), suggesting a possible role of VPE-mediated proteolysis during tapetal PCD. However, the molecular mechanisms for the dynamic reorganization of the vacuoles during tapetal PCD have not yet been elucidated.

\subsection{Conclusions and Future Perspectives}

Vacuolar collapse seems to be important in the triggering of plant PCD during innate immunity and development in both reproductive and vegetative tissues. Recent findings have shed light on the machinery for various plant PCD. Further live cell imaging may reveal novel dynamic aspects of the vacuole. In light of these circumstances, automated microscopy and image analysis techniques for the quantitative evaluation of cellular dynamics are of increasing importance.

Besides apoptosis, autophagy is also a participant in cell degeneration and PCD in animals (Shimizu et al. 2004; Tsujimoto and Shimizu 2005). Autophagic cell death is characterized by the occurrence of double-membrane autophagosomes within the dying cells that remove the cell remnants (Gump and Thorburn 2011). In Drosophila melanogaster, the destruction of the salivary glands and digestive tract was shown to be mediated by a marked upregulation of autophagy before and during cell death during metamorphosis (Melendez and Neufeld 2008).

Autophagy has recently been shown to be involved in various processes such as recycling of nutrients and senescence in plants (Yoshimoto 2012). Although autophagy-deficient mutants of Arabidopsis have been reported to show a normal life cycle, autophagy may also play roles in PCDs in development or stress responses in plants (van Doorn and Woltering 2010). A simple easy method to quantitatively analyze autophagic fluxes has recently been developed in plants (Hanamata et al. 2013). Such technical advances in combination with genetic analyses may reveal novel aspects of autophagy in PCD in plants.

Open Access: This article is distributed under the terms of the Creative Commons Attribution Noncommercial License which permits any noncommercial use, distribution, and reproduction in any medium, provided the original author(s) and source are credited. 


\section{References}

Ariizumi T, Toriyama K (2011) Genetic regulation of sporopollenin synthesis and pollen exine development. Annu Rev Plant Biol 62:437-460

Aya K, Ueguchi-Tanaka M, Kondo M et al (2009) Gibberellin modulates anther development in rice via the transcriptional regulation of GAMYB. Plant Cell 21:1453-1472

Bosch M, Franklin-Tong VE (2008) Self-incompatibility in Papaver: signalling to trigger PCD in incompatible pollen. J Exp Bot 59:481-490

Bozhkov PV, Lam E (2011) Green death: revealing programmed cell death in plants. Cell Death Differ 18:1239-1240

Cacas JL (2010) Devil inside: does plant programmed cell death involve the endomembrane system? Plant Cell Environ 33:1453-1473

Cheng H, Qin L, Lee S et al (2004) Gibberellin regulates Arabidopsis floral development via suppression of DELLA protein function. Development (Camb) 131:1055-1064

Drerup M, Schlücking K, Hashimoto K et al (2013) The calcineurin B-like calcium sensors CBL1 and CBL9 together with their interacting protein kinase CIPK26 regulate the Arabidopsis NADPH oxidase RBOHF. Mol Plant 6:559-569

Fuchs Y, Steller H (2011) Programmed cell death in animal development and disease. Cell 147:742-758

Greenberg JT (1996) Programmed cell death: a way of life for plants. Proc Natl Acad Sci USA 93:12094-12097

Gross P, Julius C, Schmelzer E et al (1993) Translocation of cytoplasm and nucleus to fungal penetration sites is associated with depolymerization of microtubules and defense gene activation in infected, cultured parsley cells. EMBO J 12:1735-1744

Gump JM, Thorburn A (2011) Autophagy and apoptosis: what is the connection? Trends Cell Biol 21:387-392

Gunawardena AH (2008) Programmed cell death and tissue remodelling in plants. J Exp Bot 59:445-451

Guo WJ, Ho TH (2008) An abscisic acid-induced protein, HVA22, inhibits gibberellin-mediated programmed cell death in cereal aleurone cells. Plant Physiol 147:1710-1722

Hanamata S, Kurusu T, Okada M et al (2013) In vivo imaging and quantitative monitoring of autophagic flux in tobacco BY-2 cells. Plant Signal Behav 8:e22510

Hara-Nishimura I, Hatsugai N (2011) The role of vacuole in plant cell death. Cell Death Differ 18:1298-1304

Hatsugai N, Kuroyanagi M, Yamada K et al (2004) A plant vacuolar protease, VPE, mediates virus-induced hypersensitive cell death. Science 305:855-858

Hatsugai N, Kuroyanagi M, Nishimura M et al (2006) A cellular suicide strategy of plants: vacuole-mediated cell death. Apoptosis 11:905-911

Hatsugai N, Iwasaki S, Tamura K et al (2009) A novel membrane fusion-mediated plant immunity against bacterial pathogens. Genes Dev 23:2496-2506

Heath MC (2000) Hypersensitive response-related death. Plant Mol Biol 44:321-334

Higaki T, Goh T, Hayashi T et al (2007) Elicitor-induced cytoskeletal rearrangement relates to vacuolar dynamics and execution of cell death: in vivo imaging of hypersensitive cell death in tobacco BY-2 cells. Plant Cell Physiol 48:1414-1425

Higaki T, Kadota Y, Goh T et al (2008) Vacuolar and cytoskeletal dynamics during elicitor-induced programmed cell death in tobacco BY-2 cells. Plant Signal Behav 3:700-703

Higaki T, Kurusu T, Hasezawa $S$ et al (2011) Dynamic intracellular reorganization of cytoskeletons and the vacuole in defense responses and hypersensitive cell death. J Plant Res 124: $315-324$

Jones AM (2001) Programmed cell death in development and defense. Plant Physiol 125:94-97

Kadota Y, Kuchitsu K (2006) Regulation of elicitor-induced defense responses by $\mathrm{Ca}^{2+}$ channels and cell cycle in tobacco BY-2 cells. In: Nagata T, Matsuoka K, Inze D (eds) Biotechnology in 
agriculture and forestry 58 tobacco BY-2 cells: from cellular dynamics to omics. Springer, Berlin, pp 207-221

Kadota Y, Goh T, Tomatsu H et al (2004a) Cryptogein-induced initial events in tobacco BY-2 cells: pharmacological characterization of molecular relationship among cytosolic $\mathrm{Ca}^{2+}$ transients, anion efflux and production of reactive oxygen species. Plant Cell Physiol 45:160-170

Kadota Y, Watanabe T, Fujii S et al (2004b) Crosstalk between elicitor-induced cell death and cell cycle regulation in tobacco BY-2 cells. Plant J 40:131-142

Kadota Y, Watanabe T, Fujii S et al (2005) Cell cycle dependence of elicitor-induced signal transduction in tobacco BY-2 cells. Plant Cell Physiol 46:156-165

Kadota Y, Fujii S, Ogasawara Y et al (2006) Continuous recognition of the elicitor signal for several hours is prerequisite for induction of cell death and prolonged activation of signaling events in tobacco BY-2 cells. Plant Cell Physiol 47:1337-1342

Kawanabe T, Ariizumi T, Kawai-Yamada M et al (2006) Abolition of the tapetum suicide program ruins microsporogenesis. Plant Cell Physiol 47:784-787

Kimura S, Kaya H, Kawarazaki T et al (2012) Protein phosphorylation is a prerequisite for the $\mathrm{Ca}^{2+}$-dependent activation of Arabidopsis NADPH oxidases and may function as a trigger for the positive feedback regulation of $\mathrm{Ca}^{2+}$ and reactive oxygen species. Biochim Biophys Acta 1823:398-405

Kimura S, Kawarazaki T, Nibori $\mathrm{H}$ et al (2013) The CBL-interacting protein kinase CIPK26 is a novel interactor of Arabidopsis NADPH oxidase AtRbohF that negatively modulates its ROSproducing activity in a heterologous expression system. J Biochem (Tokyo) 153:191-195

Kobayashi I, Kobayashi Y, Hardham AR (1994) Dynamic reorganization of microtubules and microfilaments in flax cells during the resistance response to flax rust infection. Planta (Berl) 195:237-247

Kobayashi Y, Kobayashi I, Funaki Y et al (1997) Dynamic reorganization of microfilaments and microtubules is necessary for the expression of non-host resistance in barley coleoptile cells. Plant J 11:525-537

Kobayashi M, Ohura I, Kawakita K et al (2007) Calcium-dependent protein kinases regulate the production of reactive oxygen species by potato NADPH oxidase. Plant Cell 19:1065-1080

Koh S, Andre A, Edwards H et al (2005) Arabidopsis thaliana subcellular responses to compatible Erysiphe cichoracearum infections. Plant J 44:516-529

$\mathrm{Ku} \mathrm{S}$, Yoon H, Suh HS et al (2003) Male-sterility of thermosensitive genic male-sterile rice is associated with premature programmed cell death of the tapetum. Planta (Berl) 217:559-565

Kuriyama H (1999) Loss of tonoplast integrity programmed in tracheary element differentiation. Plant Physiol 121:763-774

Kutsuna N, Hasezawa S (2002) Dynamic organization of vacuolar and microtubule structures during cell cycle progression in synchronized tobacco BY-2 cells. Plant Cell Physiol 43:965-973

Lecourieux D, Mazars C, Pauly N et al (2002) Analysis and effects of cytosolic free calcium increases in response to elicitors in Nicotiana plumbaginifolia cells. Plant Cell 14:2627-2641

Li N, Zhang DS, Liu HS et al (2006) The rice tapetum degeneration retardation gene is required for tapetum degradation and anther development. Plant Cell 18:2999-3014

Marty F (1999) Plant vacuoles. Plant Cell 11:587-600

Melendez A, Neufeld TP (2008) The cell biology of autophagy in metazoans: a developing story. Development (Camb) 135:2347-2360

Mur LA, Kenton P, Lloyd AJ et al (2008) The hypersensitive response; the centenary is upon us but how much do we know? J Exp Bot 59:501-520

Nakaune S, Yamada K, Kondo M et al (2005) A vacuolar processing enzyme, $\delta$ VPE, is involved in seed coat formation at the early stage of seed development. Plant Cell 17:876-887

Obara K, Kuriyama H, Fukuda H (2001) Direct evidence of active and rapid nuclear degradation triggered by vacuole rupture during programmed cell death in Zinnia. Plant Physiol 125:615-626

Ogasawara Y, Kaya H, Hiraoka G et al (2008) Synergistic activation of Arabidopsis NADPH oxidase AtrbohD by $\mathrm{Ca}^{2+}$ and phosphorylation. J Biol Chem 283:8885-8892 
Ohno R, Kadota Y, Fujii S et al (2011) Cryptogein-induced cell cycle arrest at $\mathrm{G}_{2}$ phase is associated with inhibition of cyclin-dependent kinases, suppression of expression of cell cycle-related genes and protein degradation in synchronized tobacco BY-2 cells. Plant Cell Physiol 52:922-932

Pennell RI, Lamb C (1997) Programmed cell death in plants. Plant Cell 9:1157-1168

Reape TJ, McCabe PF (2010) Apoptotic-like regulation of programmed cell death in plants. Apoptosis 15:249-256

Rogers LA, Dubos C, Surman C et al (2005) Comparison of lignin deposition in three ectopic lignification mutants. New Phytol 168:123-140

Saito C, Ueda T, Abe H et al (2002) A complex and mobile structure forms a distinct subregion within the continuous vacuolar membrane in young cotyledons of Arabidopsis. Plant J 29:245-255

Shimizu S, Kanaseki T, Mizushima N et al (2004) Role of Bcl-2 family proteins in a non-apoptotic programmed cell death dependent on autophagy genes. Nat Cell Biol 6:1221-1228

Skalamera D, Heath MC (1996) Cellular mechanisms of callose deposition in response to fungal infection or chemical damage. Can J Bot 74:1236-1242

Smertenko AP, Bozhkov PV, Filonova LH et al (2003) Re-organisation of the cytoskeleton during developmental programmed cell death in Picea abies embryos. Plant J 33:813-882

Suzuki N, Miller G, Morales J et al (2011) Respiratory burst oxidases: the engines of ROS signaling. Curr Opin Plant Biol 14:691-699

Takeda S, Gapper C, Kaya H et al (2008) Local positive feedback regulation determines cell shape in root hair cells. Science 319:1241-1244

Takemoto D, Jones DA, Hardham AR (2003) GFP-tagging of cell components reveals the dynamics of subcellular re-organization in response to infection of Arabidopsis by oomycete pathogens. Plant J 33:775-792

Teng X, Cheng WC, Qi B et al (2011) Gene-dependent cell death in yeast. Cell Death Dis 2:e188

Torres MA, Jones JD, Dangl JL (2005) Pathogen-induced, NADPH oxidase-derived reactive oxygen intermediates suppress spread of cell death in Arabidopsis thaliana. Nat Genet 37: $1130-1134$

Tsujimoto Y, Shimizu S (2005) Another way to die: autophagic programmed cell death. Cell Death Differ 12(2):1528-1534

van Doorn WG, Woltering EJ (2010) What about the role of autophagy in PCD? Trends Plant Sci 15:361-362

Vardar F, Unal M (2012) Ultrastructural aspects and programmed cell death in the tapetal cells of Lathyrus undulatus Boiss. Acta Biol Hung 63:52-66

Vizcay-Barrena G, Wilson ZA (2006) Altered tapet al PCD and pollen wall development in the Arabidopsis ms1 mutant. J Exp Bot 57:2709-2717

Wan L, Xia X, Hong D et al (2010) Abnormal vacuolization of the tapetum during the tetrad stage is associated with male sterility in the recessive genic male sterile Brassica napus L. line 9012A. J Plant Biol 53:121-133

Wang M, Hoekstra S, Van-Bergen S et al (1999) Apoptosis in developing anthers and the role of ABA in this process during androgenesis in Hordeum vulgare L. Plant Mol Biol 39:489-501

Wei CX, Lan SY, Xu ZX (2002) Ultrastructural features of nucleus degradation during programmed cell death of starchy endosperm cells in rice. Acta Bot Sin 44:1396-1402

Wright H, van Doorn WG, Gunawardena AH (2009) In vivo study of developmental programmed cell death using the lace plant (Aponogeton madagascariensis; Aponogetonaceae) leaf model system. Am J Bot 96:865-876

Wu H, Yang M (2005) Reduction in vacuolar volume in the tapetal cells coincides with conclusion of the tetrad stage in Arabidopsis thaliana. Sex Plant Reprod 18:173-178

Yoshimoto K (2012) Beginning to understand autophagy, an intracellular self-degradation system in plants. Plant Cell Physiol 53:1355-1365

Zhang DS, Liang WQ, Yuan Z et al (2008) Tapetum degeneration retardation is critical for aliphatic metabolism and gene regulation during rice pollen development. Mol Plant 1:599-610 